Approvedfor public release; distribution is unlimited
Title: A POSTERIORI ERROR DETERMINATION AND GRID ADAPTATION
=OR AMR AND ALE COMPUTATIONAL FLUID DYNAMICS

Author(s) : Giovanni Lapenta

Submitted:

Computational Fluid Dynamics

Sydney

15-19 July, 2002

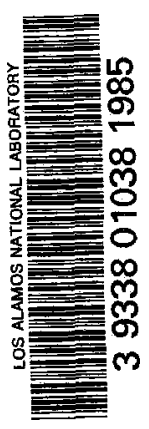

Group

Address

Plasma Theory Group
Los Alamos National Laboratory
Los Alamos, New Mexico 87545

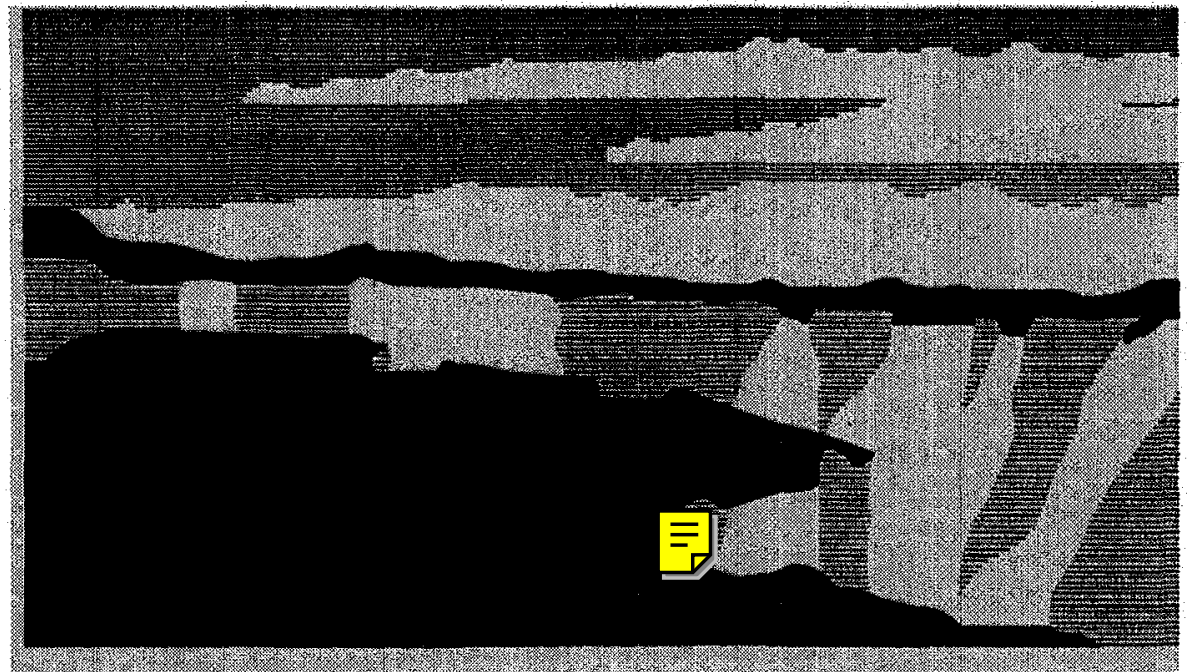

Los Alamos National Laboratory, an affirmative action/equal opportunity employer, is operated by the University of Callfornla for the U.S. Department of Energy under contract W.7405-ENG-36. By acceptance of this article, the publlsher recognizes that the U.S. Government retains a nonexclusive, royalty-tree license to publish or reproduce the publishedform of this contribution, or to allow others to do so, for U.S. Government purposes. Los Alamos National Laboratory requests that the publisher identify this article as work performed under the auspices of the U.S. Department of Energy. The Los Alamos National Laboratory strongly supports academic freedom and a researcher's right to publish; as an institution, however, the Laboratory does not endorse the viewpoint of a publicationor guarantee it's technical correctness. 


\title{
A Posteriori Error Determination and Grid Adaptation for AMR and ALE Computational Fluid Dynamics
}

\author{
Giovanni Lapenta \\ Theoretical Division, Los Alamos National Laboratory, Los Alamos NM87545, USA
}

\begin{abstract}
We discuss grid adaptation for application to AMR and ALE codes. Two new contributions are presented. First, a new method to locate the regions where the truncation error is being created clue to an insufficient accuracy: the operator recovery error origin (OREO) detector. The OREO detector is automatic, reliable, easy to implement and extremely inexpensive. Second, a new grid motion technique is presented for application to ALE codes. The method is based. on the BrackbillSaltzman approach but it is directly linked to the OREO detector and moves the grid automatically to minimize the error.
\end{abstract}

\section{Introduction}

Grid adaptation can be achieved by grid refinement (i.e. adding more grid points) in some selected areas or by grid motion (i.e. moving grid points to regions of interest from regions of lesser interest). In the first, case, the AMR method [2] is obtained. In the second case, the ALE method [4] is obtained. In both cases we need guidance. We need to know what interesting mean. Often, interest is defined based on the knowledge of the solution. If we know the solution, we know where more accuracy is required. However, we do not, in general, know the solution. So we need error detectors which tell us where the error is larger.

The present paper describes a new error detector: the operator recovery error origin (OREO) detector [6].

For AMR codes, the OREO detector provides accurate and automatic determination of where the discretization error is being generated. This knowledge is directly used by the AMR method to refine or to coarsen.

For ALE codes, the knowledge of the error needs to be supplemented by a method to move the grid. Given the error what, new grid should we use? To answer this additional problem typical of the ALE method we also present a new technique citelapenta based on the Brackbill-Saltzman approach [8].

Examples are presented to illustrate the new methods.

\section{Operator Recovery Computation of the Local Truncation Error}

In a previous paper [6], we have proposed a new error origin detector based on the extension of the gradient recovery error estimator [1]. We have named the 
approach operator recovery error origin (OREO) detector since it extends to any operator the method used for the gradient operator by the gradient recovery error estimator. Below, we summarize briefly the procedure involved in its definition and implementation.

For the sake of definiteness, we shall assume a general $\mathrm{N}$-dimensional grid (where one of the dimensions could be time) where a vector field $\mathrm{v}_{n}$ is node centered. For notation, we label the cells with $c$ and the nodes with $n$, using further the notation $n(c)$ to indicate the nodes neighboring cell $c$ and $c(n)$ to indicate the cells neighboring node $n$.

We consider a general multi-dimensional non-linear partial differential operator:

$$
\mathbb{Q}(q)
$$

Equation (1) summarizes the most general operator acting on a function $q(\mathrm{x})$ defined on the multidimensional space $\mathbf{x}$.

Equation (1)is discretized on a grid with $N$ nodes $\mathbf{x}_{n}$ :

$$
O_{n}\left(q_{1}, \ldots, q_{N}\right)
$$

From the discretized field $q_{n}$ and from the discretized operator $X_{n}$ applied to $q_{n}$ defined only on the grid nodes, it is possible to reconstruct two functions defined everywhere in the continuum space $\mathbf{x}$ :

$$
\begin{aligned}
& \tilde{q}(\mathbf{x})=\mathcal{L}\left\{q_{n}\right\} \\
& \widetilde{O}(\mathbf{x})=\mathcal{L}\left\{O_{n}\left(q_{1}, \ldots, q_{N}\right)\right\}
\end{aligned}
$$

where $\mathcal{L}$ is the operator representing the multilinear (e.g., linear in 1D, bilinear in 2D) interpolation from the grid points.

The local truncation error is defined as the difference between the linear interpolation of the discretized operator applied to the discretized field $\widetilde{X_{q}}(\mathbf{x})$ and the exact differential operator applied to the linear interpolation of the discretized field $\tilde{q}(\mathbf{x})$ :

$$
e=\tilde{O}(\mathrm{x})-\mathfrak{O} \tilde{q}(\mathbf{x})
$$

The average local truncation error on any given cell $c$ is defined as the $L_{2}$ norm:

$$
e_{c}=\left(\frac{1}{V_{c}} \int_{V_{c}} e^{2} d V\right)^{1 / 2}
$$

where e, is the average local truncation error over cell $c$ and $V_{c}$ is the cell volume.

\section{Variational Grid Adaptation Based on Error Indicators}

We have recently proposed a new approach [5] to variational grid adaptation [8] based of the local truncation error defined above. The method can be constructed starting from the following equidistribution theorem proven in Ref. [5] 
THEORTM: In a optimal grid, defined as a grid that minimizes the local truncation error according to the minimzation principle

$$
\int_{\mathcal{V}}|e| d^{N} x
$$

the product of the local truncation error in any cell $i$ by the cell volume $V_{i}$ (given by the Jacobian $J=\sqrt{g}$ ) is constant:

$$
e_{i} V_{i}=\text { const }
$$

The equidistribution theorem is applied solving the following Euler-Lagrange equations:

$$
g^{i j} \frac{\partial}{\partial \xi^{i}}\left(|e| \frac{\partial x^{i}}{\partial \xi^{j}}\right)=0
$$

This approach creates a grid where $\left|e_{i}\right| V_{i}$ is constant. Note that the equations above are identical to the equations used by the Brackbill-Saltzman variable diffusion method [8]. The primary innovation is that the monitor function is now directly linked with the local truncation error instead of being left undefined. In the typical implementations of the Brackbill-Saltzman method, the monitor Etinction is defined heuristically by the user. The use of the OREO detector proposed here results in a more accurate scheme [5].

\section{ALE Example}

We have applied the grid rezoning described above to a standard ALE code $[4,7]$ and we have tested it in a number of problems. Here we limit the discussion to the classic spherical 1D implosion test proposed by Noli [9]. A gas with $\gamma=5 / 3$ initially has $\rho=1, e=10^{-4}$ and uniform velocity $u=-1$ (except in the center where $u(r=0)=0$ ). The problem represents a serious challenge for Lagrangian calculations and the solution is known to suffer from serious wall heating due to the use of artificial viscosity to capture shocks. Note that we are not using artificial heat conduction [9] (a tool to mitigate the wall heating problem) precisely to highlight the trouble of Lagrangian calculations for the present case .

The results of an ALE calculation using the adaptive grid is compared with a reference standard Lagrangian calculation. Figure 1 shows the density at the end of the Lagrangian and ALE calculation. The use of adaptive grid results in a much improved solution. The reason for the improvement is explained by the sharper resolution of the shock achieved by the adaptation. As noted in the original paper by Noli [9], a sharper resolution of the shock also implies a reduction of wall heating, as obseved in Fig. 1 for the ALE case.

The use of grid adaptation based on the OREO detector results in an automatic method to increase the accuracy of the ALE method. 


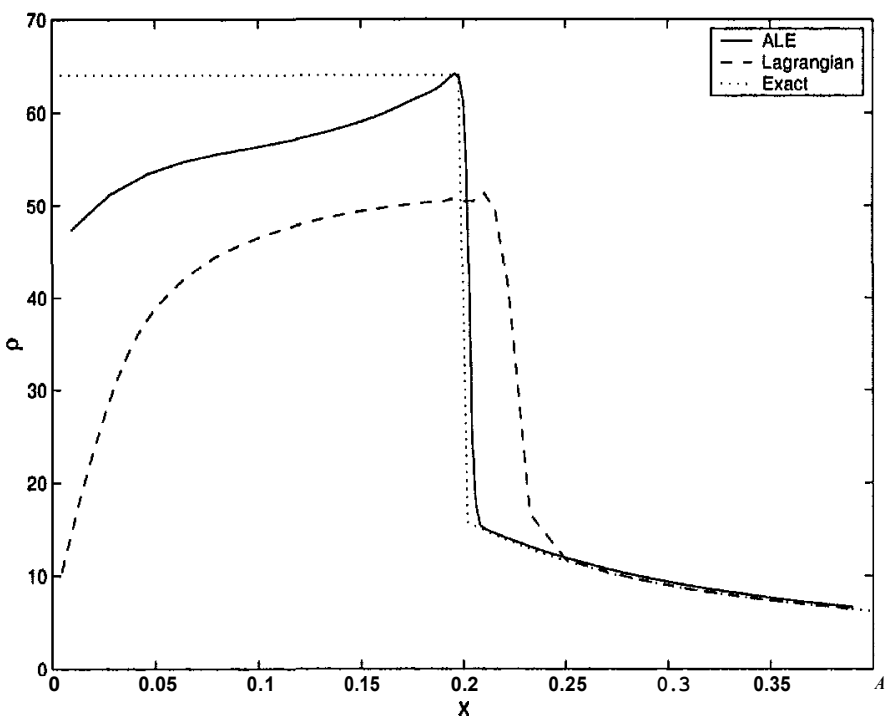

Fig. 1. Noh's spherical benchmark: comparison of the density at the end $(t=0.6)$, for a Lagrangian (dashed) and ALE (solid line) calculation. The exact solution is also shown (dotted line).

\subsection{AMR Example}

To investigate the performance of the OREO detector in 2D, we have applied it to results obtained with CLAWPACK [10]. CLAWPACK is a publicly available software [11] based on an AMR solution [2] of the conservation laws. We have applied the code to the solution of the gas dynamics equations for the Colella's wedge problem [3]. A planar $M=10$ shock is incident on an oblique surface; the angle between the shock direction and the surface is $\pi / 6$. The actual computed results at time $t=0.2$ for a $240 \times 120$ grid are shown in Fig. 2 where all the expected features [3] can be recognized.

The OREO detector is computed based on the results obtained from CLAWPACK using Algorithm 3. The detector is shown in Fig. 3 for a simulation with a grid $120 \times 60$. For comparison we also provide an estimate of the actual error, computed by difference between the solution on a $120 \times 60$ grid and the more accurate solution on a $240 \times 120$ grid. Clearly the OREO detector is successful in detecting all origins of errors. The shocks are all captured; the slip surface rolling up under the shock is evident. All features are detected.

For reference, Fig. 3-c shows also a similar analysis conducted on another possible candidate for error detection often used in the literature. The detector, which we name warp indicator for convenience, measure the local error as the variance among the different values obtained at a node when extrapolating the internal energy from the four directions (backward and forward along $x$ and 
backward and forward along $y$ ). The analysis in Fig. 3-c shows that the two rightmost planar shocks are captured well, while the top and bow shocks are barely visible. All the structine inside the rolling up region within the outer shocks is lost: no slip surface is measured and the internal shock is also lost. In practice the warp indicator is often supplemented by other ad hoc detectors to pick up all shocks, but still the rolling up region and the slip surfaces are often Jeli undetected.

The ORTEO detector doos not miss any feature and can bo used reliably alone without any other ad hoc detector.
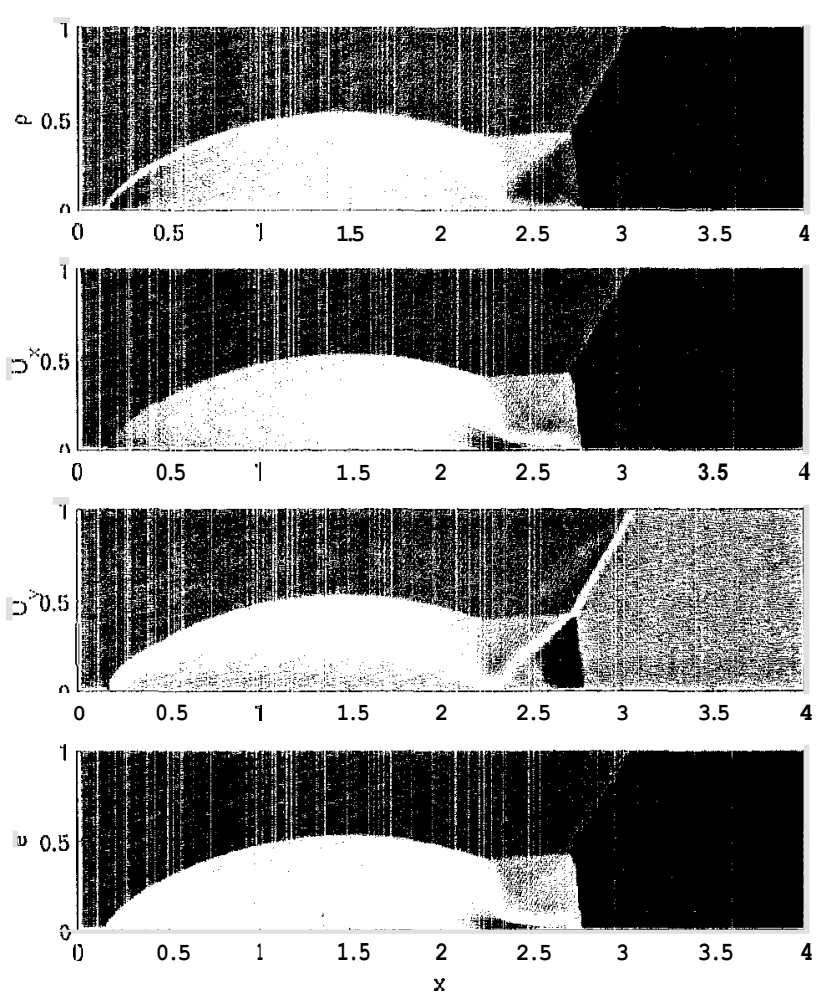

Tig. 2. 21) Gas Dynamics (Fulerian form)- Colella's benchmark on a 240x120grid. Donsity, velocity and intomal energy at the ond of a Jsulerian calculation $(t=0.2)$. Rosults obtained using CLAWPACK. 

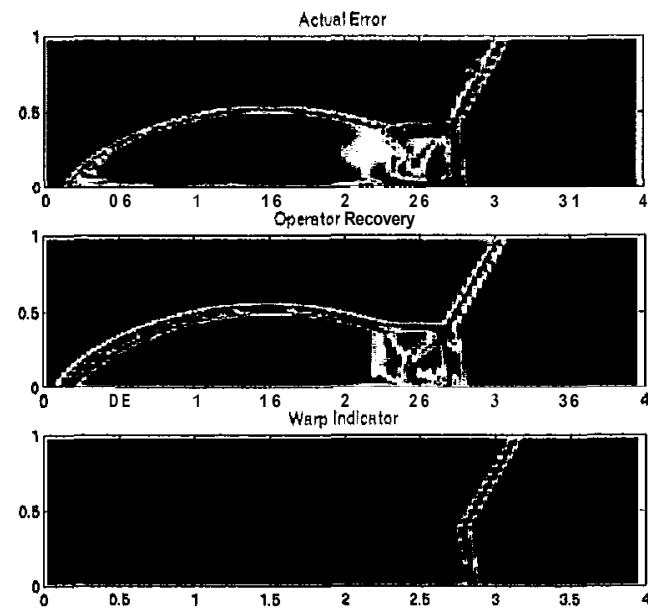

Fig. 3. 2D Gas Dynamics - Colella's benchmark on a 120x60 grid at $t=\mathbf{0 . 2}$. Comparison of the global truncation error (a) with the OREO detector (b), and the warp indicator $(c)$.

\section{Acknowledgments}

The author wishes to acknowledge many useful discussions on the topics reported in the present manuscript with Jerry Brackbill, Doug Kothe, Alex Kurganov, Len Margolin, Tony Scannapieco and Misha Shashkov. This research is supported by the National Nuclear Security Administration under the Advance Strategic Computing Initiative (ASCI) Program.

\section{References}

1. M. Ainsworth, J.T. Oden, A Posteriori Error Estimation in Finite Element Analysis (Wiley, New York, 2000).

2. M.J. Berger, J. Oliger, J. Comprtt. Phys., 63, 484(1984)

3. P. Colella., J. Comput. Phys., 87, 171 (1990)

4. C.W. Hirt, A.A. Amsden, J.L. Cook, .J. Comp. Phys., 14, 227 (1974).

5. G. Lapenta, J. Comput. Phys., submitted.

6. G. Lapenta, Int. J. Num. Meth Engnrg, submitted.

7. G. Lapenta, "Grid Adaptation and Remapping for Arbitrary Lagrangian Enlerian (ALE) Methods", Proceedings of the 8th International Conference on Numerical Grid Generation in Compistational Field Simulations, Honolulu, June 2-6, (2002).

8. J.U. Brackbill, J. Comput. Phys., 108, 38 (1993).

9. W.F. Noh, J. Comput. Phys, 72, 78 (1987).

10. R.J. LeVeque, CLAWPACK Version 4.O User's Manual (University of Washington, 1999).

11. http://www.amath.washington.edu/ claw/ 